\title{
Prediction of general hospital admission in people with dementia: cohort study
}

\author{
Tom C. Russ, Mario A. Parra, Alison E. Lim, Emma Law, Peter J. Connelly and John M. Starr
}

\section{Background}

People with dementia are extremely vulnerable in

hospital and unscheduled admissions should be avoided if possible.

\begin{abstract}
Aims
To identify any predictors of general hospital admission in people with dementia in a well-characterised national prospective cohort study.
\end{abstract}

\section{Method}

A cohort of 730 persons with dementia was drawn from the Scottish Dementia Research Interest Register (47.8\% female; mean age 76.3 years, s.d. $=8.2$, range $50-94$ ), with a mean follow-up period of 1.2 years.

\section{Results}

In the age- and gender-adjusted multivariable model $(n=681$ 251 admitted), Neuropsychiatric Inventory score (hazard ratio per s.d. disadvantage $1.21,95 \% \mathrm{Cl} 1.08-1.36$ ) was identified as an independent predictor of admission to hospital.

\section{Conclusions}

Neuropsychiatric symptoms in dementia, measured using the Neuropsychiatric Inventory, predict non-psychiatric hospital admission of people with dementia. Further studies are merited to test whether interventions to reduce such symptoms might reduce unscheduled admissions to acute hospitals.

\section{Declaration of interest}

None.
Dementia is a major and increasing public health concern. There are currently over 800000 people with dementia in the UK, ${ }^{1}$ and approximately $6 \%$ of these individuals are estimated to be in hospital at any one time, ten times the rate in older adults without dementia. ${ }^{2}$ People with dementia in acute general hospital wards are extremely vulnerable and suffer from increased mortality, ${ }^{3-5}$ as well as other adverse outcomes including longer length of stay, increased rates of delirium and new institutionalisation. ${ }^{6}$ There is general agreement that unscheduled admission to hospital should be avoided or prevented in this group, where possible. ${ }^{7,8}$ However, little attention has been paid to the factors which might influence the risk of a person with dementia being admitted to hospital, as highlighted by a recent systematic review on the topic. ${ }^{9}$ Candidate risk factors include age, gender, function, behaviour, medication use and comorbidity. ${ }^{9-13}$ Published studies on risk of admission are either based on clinical samples, ${ }^{10,12}$ which limits the generalisation of their findings to the general population, or are limited in size, duration of follow-up or the detail in which participants are characterised at baseline. ${ }^{11-14}$ Here we present a prospective cohort study of the association between baseline health, cognitive and functional status and admission to general hospital in a well-characterised national sample of community-dwelling older adults with dementia from across Scotland.

\section{Method}

The Scottish Dementia Research Interest Register (SDRIR) comprises people from a variety of demographic backgrounds who have been diagnosed by a clinician as having dementia or a related cognitive disorder (and carers, who were not included in this study) and who have consented (or in cases where the person lacked capacity, through his or her legal representative) to the storage of information on demographic, cognitive, functional and behavioural measures and have expressed an interest in being approached for participation in future research studies. ${ }^{15}$ At the time of this study people had been enrolled from eight areas of
Scotland covering $75 \%$ of the Scottish population including both urban and rural areas. Additional details of the SDRIR and recruitment to it are available in a published report. ${ }^{15}$ Approximately $95 \%$ of SDRIR registrants consented to record linkage.

\section{Assessment}

An individual joining the register is visited by a clinical studies officer who assesses and records cognition using the Addenbrooke's Cognitive Examination - Revised (ACE-R). ${ }^{16}$ Function and behaviour are rated, through interviewing the carer, using the Instrumental Activities of Daily Living (IADL) scale, the Physical Self-Maintenance Scale (PSMS) and the Neuropsychiatric Inventory (NPI) including the caregiver distress scale. ${ }^{17,18}$ A global assessment of severity, the Clinical Dementia Rating scale (CDR), is completed by interviewing the patient and carer. ${ }^{19,20}$ The ACE-R is scored out of 100 and the forms used allow direct calculation of the Mini-Mental State Examination (MMSE) score. ${ }^{21}$ The IADL and PSMS are scored out of 8 and 6 respectively, with one point awarded for each domain of activity in which the individual can function independently; higher scores indicate greater independence. The NPI was recorded as the product of frequency and severity scores for each domain (ranging from 1 to 12) with caregiver distress rated separately. ${ }^{22}$ Total NPI and caregiver distress scores are the sum of scores for all ten domains. The CDR consists of global ratings in six domains or 'boxes' (memory, orientation, judgement/problem-solving, community affairs, home and hobbies, and personal care) with a rating of 0 (none), 0.5 (very mild), 1 (mild), 2 (moderate) or 3 (severe) being given for each. ${ }^{19}$ Details of any illnesses or conditions that are also present and prescribed medication are recorded, as are health-related behaviours (including smoking) and the number of carers. To overcome coding problems associated with initial data entry into the SDRIR and to correct obvious misclassifications (primarily 'young-onset dementia' and 'early dementia'), clinical diagnoses were independently verified by two clinicians (two of T.C.R., P.J.C. and J.M.S.) reviewing the data recorded on the SDRIR with 
disagreement resolved by discussion or, if necessary, consultation with the third clinician. The medications recorded for each person were scrutinised and individuals prescribed cholinesterase inhibitors or antipsychotics were separately flagged. Additionally, any potentially inappropriate medication for use in older adults was identified according to the updated Beers criteria. ${ }^{23}$

Informed consent for potential data linkage was obtained from all participants at the time of registration on the SDRIR or, if they were incapable of consent, from their legal representative. Ethical approval for the SDRIR and the data linkage was granted by the Scotland A Research Ethics Committee and the record linkage was approved by the Privacy Advisory Committee of the Information Services Division of National Health Service (NHS) National Services Scotland.

\section{Statistical analysis}

Data from consenting registrants on the SDRIR were linked, using the unique Community Health Index number, with Scottish Morbidity Records 01 and 04 which respectively record all admissions to general and psychiatric hospitals in Scotland. The date of the admission and all diagnostic codes recorded on admission and discharge were recorded. For this analysis admission to a general hospital was the outcome of interest; data for individuals admitted to a psychiatric hospital were considered censored at that point. The SDRIR data are held by the Health Informatics Centre (HIC) at the University of Dundee and the anonymised, linked data-set was accessed through and analysed in the HIC safe haven using IBM SPSS Statistics version 21.

We calculated the time in calendar days from the date of entry to the SDRIR until the first admission to a general hospital for any reason. For people who were not admitted to hospital, data were censored either at 2 June 2012 or at the date of admission to a psychiatric hospital $(n=25)$, whichever came first. After confirming that the proportional hazards assumption was valid, we used Cox regression models to produce hazard ratios with accompanying 95\% confidence intervals for the association between individual baseline covariables and admission to hospital. ${ }^{24}$ Covariables examined comprised dementia subtype, the presence of vascular risk factors or comorbidity, having a carer, MMSE, IADL and PSMS scores, total NPI score, NPI caregiver distress score, CDR rating and CDR sum of boxes. Given the possibility that cholinesterase inhibitor use may be associated with reduced risk of entry to nursing home care,${ }^{25}$ and the concerns surrounding the use of antipsychotic drugs, ${ }^{26}$ these variables were also included, as was being prescribed a potentially inappropriate medication. For this analysis continuous variables were standardised so that hazard ratios reflected 1 s.d. disadvantage regardless of the original direction of the scale. There was no effect modification by gender and therefore data for men and women were pooled. All models included age and gender as these were thought to be of importance a priori. Next, having considered bivariate associations, we noted that some variables were moderately correlated (for example, cognition and activities of daily living). Since we wished to include the key independent variables predicting hospital admission we constructed a multivariable model using forward stepwise entry.

A recent systematic review suggested that the NPI might be important in predicting admission to hospital, ${ }^{9}$ and so a number of sensitivity analyses examining this association in more detail were carried out. First, we classified total NPI score into three categories, based on published recommended thresholds, ${ }^{27}$ in order to examine the shape of the relationship between NPI and hospital admission; second, we investigated the association between individual items and admission to hospital. Finally, individuals with complete data for all variables were compared with individuals with missing data for one or more variable. Missing data were accounted for by repeating the multivariable model using five multiply imputed data-sets.

\section{Results}

From an initial sample of 762 we excluded 26 individuals with mild cognitive impairment and 6 individuals with data errors (e.g. duplicated records, missing identifiers or impossible dates of birth), giving an analytic sample of 730 (47.8\% female; mean age 76.3 years, s.d. $=8.2$, range $50-94$ ). Figure 1 summarises the derivation of the analytic sample. During a follow-up period of 1.2 years (s.d. $=0.8$, range 2 days to 3.3 years), $274(37.5 \%)$ of the 730 SDRIR registrants were admitted to a general hospital for any reason. Online Table DS1 summarises the number of people who had various common conditions mentioned in their records on discharge from hospital, including falls or collapse $(15.3 \%)$, ischaemic heart disease $(10.6 \%)$ and urinary tract infection (10.2\%). Out of 274 admissions, 146 (53.3\%) had dementia correctly recorded on discharge, although 6 of these were recorded as having mild cognitive disorder (ICD-10 code F06.7). ${ }^{28}$ In contrast, all of the 25 individuals admitted to a psychiatric hospital (not included as admissions in the current analyses) had dementia correctly recorded on their discharge documentation.

Baseline characteristics of the sample are shown in Table 1, stratified into those who were admitted to hospital and those who were not. Just under half of the participants were women, the majority had mild to moderate dementia at baseline and the mean age was in the mid-70s for both groups, although the range spanned several decades. Approximately $80 \%$ had Alzheimer's disease or mixed dementia and about three-quarters were treated with a cholinesterase inhibitor.

\section{Bivariate analyses}

Men were more likely to be admitted to hospital than women $(\mathrm{HR}=1.32$, 95\% CI $1.04-1.68 ; P=0.022)$, but age was not associated with admission rates at conventional levels of statistical significance (per 5 years older $\mathrm{HR}=1.07,95 \%$ CI $0.99-1.16$; $P=0.07)$. Online Table DS2 shows the association between other baseline covariables and subsequent admission to hospital. There was no increased risk of admission for any diagnostic subtype, apart from the category of 'other dementia', incorporating

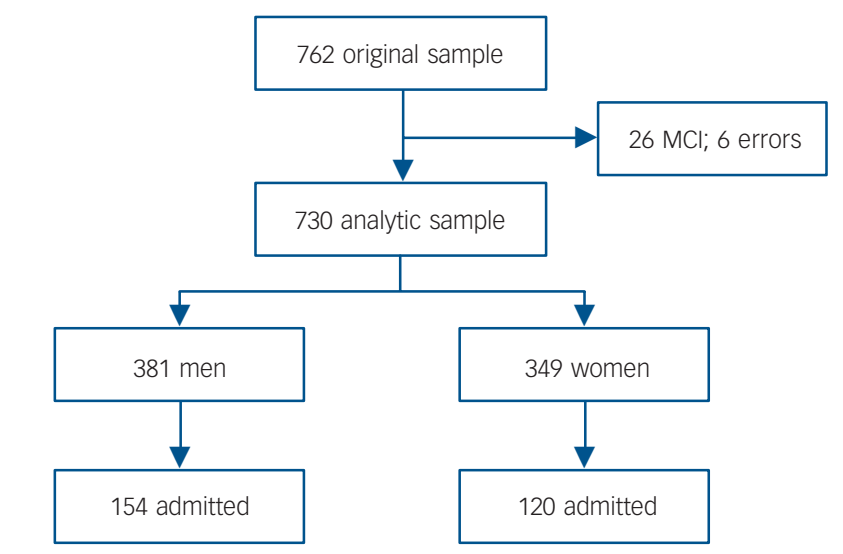

Fig. 1 Flow chart of participants from initial pooled sample through to analytic sample showing subsequent admission to hospital. $\mathrm{MCl}$, mild cognitive impairment. 
Table 1 Baseline characteristics of study participants according to whether or not an individual was admitted to hospital: Iongitudinal analysis of 730 men and women from the Scottish Dementia Research Interest Register

\begin{tabular}{|c|c|c|c|}
\hline Characteristic & Admitted to hospital $(n=274)$ & Not admitted $(n=456)$ & $P$ \\
\hline \multirow{2}{*}{$\begin{array}{l}\text { Length of follow-up, years: mean (s.d.) } \\
\text { maximum }\end{array}$} & $0.8(0.6)$ & $1.4(0.8)$ & \multirow{2}{*}{$<0.001$} \\
\hline & 2.8 & 3.3 & \\
\hline \multirow{2}{*}{$\begin{array}{l}\text { Age, years: mean (s.d.) } \\
\text { range }\end{array}$} & $76.5(8.1)$ & $76.1(8.3)$ & \multirow[t]{2}{*}{0.51} \\
\hline & 52-94 & 50-93 & \\
\hline Gender, female: $n$ (\%) & $120(43.8)$ & $229(50.2)$ & 0.09 \\
\hline \multicolumn{3}{|l|}{ Diagnosis, $n$ (\%) } & \multirow[t]{6}{*}{0.66} \\
\hline Early-onset Alzheimer's disease & $25(9.1)$ & $49(10.7)$ & \\
\hline Late-onset Alzheimer's disease & $155(56.6)$ & $264(57.9)$ & \\
\hline Mixed & $38(13.9)$ & $70(15.4)$ & \\
\hline Vascular & $32(11.7)$ & $47(10.3)$ & \\
\hline Other & $34(12.4)$ & $26(5.7)$ & \\
\hline \multirow{3}{*}{$\begin{array}{l}\text { Vascular risk factors, } n(\%)^{b} \\
\text { Any comorbidity } \\
\text { No carers }\end{array}$} & $140(51.1)$ & $236(51.8)$ & \multirow{3}{*}{$\begin{array}{c}0.03 \\
<0.001 \\
0.44\end{array}$} \\
\hline & $175(63.9)$ & $228(50.0)$ & \\
\hline & $19(6.9)$ & $29(6.4)$ & \\
\hline \multirow{3}{*}{$\begin{array}{l}\text { Prescribed cholinesterase inhibitor, } n(\%) \\
\text { Prescribed antipsychotic, } n(\%) \\
\text { Prescribed potentially inappropriate medication, } n(\%)^{d}\end{array}$} & $197(71.9)$ & $347(76.1)$ & \multirow{3}{*}{$\begin{array}{l}0.21 \\
0.65 \\
0.81\end{array}$} \\
\hline & $14(5.1)$ & $20(4.4)$ & \\
\hline & $25(9.1)$ & $44(9.6)$ & \\
\hline \multirow{6}{*}{$\begin{array}{l}\text { MMSE score, mean (s.d.) } \\
\text { IADL score, mean (s.d.) } \\
\text { PSMS score, mean (s.d.) } \\
\text { NPI total score, mean (s.d.) } \\
\text { NPI carer distress score, mean (s.d.) e } \\
\text { CDR overall rating, } n(\%)^{e}\end{array}$} & $21.3(6.2)$ & $21.3(5.9)$ & 0.94 \\
\hline & $3.5(2.3)$ & $3.7(2.3)$ & 0.27 \\
\hline & $4.1(2.0)$ & $4.3(2.0)$ & 0.23 \\
\hline & $12.2(15.7)$ & $10.9(15.2)$ & 0.30 \\
\hline & $6.4(7.9)$ & $6.3(8.0)$ & 0.87 \\
\hline & & & \multirow[t]{6}{*}{0.09} \\
\hline 0 & $7(2.7)$ & $18(4.1)$ & \\
\hline 0.5 & $104(40.2)$ & $181(41.1)$ & \\
\hline 1 & $96(37.1)$ & 165 (37.5) & \\
\hline 2 & $45(17.4)$ & $51(11.6)$ & \\
\hline 3 & $7(2.7)$ & $25(5.7)$ & \\
\hline CDR sum of values, mean (s.d.) ${ }^{e}$ & $5.8(3.8)$ & $5.7(4.0)$ & 0.85 \\
\hline \multicolumn{4}{|c|}{$\begin{array}{l}\text { a. 'Other dementia' comprises dementia with Lewy bodies, Parkinson's disease dementia, frontotemporal dementia, and other (not specified). } \\
\text { b. Vascular risk factors include hypertension, hypercholesterolaemia, diabetes and smoking. } \\
\text { c. Comorbidity includes cancer, respiratory disease, and diabetes. } \\
\text { d. Potentially inappropriate medication according to updated Beers criteria. }{ }^{22} \\
\text { e. Ratings of cognition, function and behaviour were not available for the complete sample, missing data as follows: Mini-Mental State Examination (MMSE), missing } n=44 \text {; } \\
\text { Instrumental Activities of Daily Living (IADL) scale, missing } n=67 \text {; Physical Self-Maintenance Scale (PSMS), missing } n=29 \text {; Neuropsychiatric Inventory (NPI), missing } n=34 \text {; } \\
\text { Clinical Dementia Rating (CDR) scale, overall rating missing } n=31 \text {, sum of values missing } n=53 \text {. }\end{array}$} \\
\hline
\end{tabular}

dementia with Lewy bodies, Parkinson's disease dementia, frontotemporal lobar degeneration and 'other dementia, not specified'. The suggestion that people with non-Alzheimer's, non-vascular dementia subtypes might be more likely to be admitted to hospital owing to a higher frequency of neuropsychiatric symptoms in those conditions was explored in a Cox model incorporating age and gender with total NPI score and diagnostic grouping selected by forward conditional stepwise entry. Total NPI score remained in the model (per s.d. disadvantage $\mathrm{HR}=1.22$, 95\% CI $1.09-$ 1.37; $P<0.001$ ), but having a diagnosis of 'other' dementia was not statistically significant at conventional levels in this model.

There was no statistically significant association between individual vascular risk factors (hypertension, hypercholesterolaemia, diabetes or smoking) or individual comorbidities (including cancer, respiratory disease and diabetes) and so these were separately pooled into composite variables for reporting in Table 2. Vascular risk factors were not associated with future admission to hospital, but having any comorbidity was $(\mathrm{HR}=1.28,95 \%$ CI $1.00-1.65 ; P=0.05)$. None of the three medication variables investigated - cholinesterase inhibitors, antipsychotics and potentially inappropriate medications - were associated with an increased risk of hospital admission. Baseline cognition was not associated with admission to hospital, nor was impaired functional ability as measured by the IADL scale. However, impaired self-care, measured by the PSMS, was associated with an increased risk of admission (per s.d. disadvantage $\mathrm{HR}=1.18,95 \%$ CI $1.04-1.33 ; P=0.01)$. Total NPI score $(\mathrm{HR}=1.22$, 95\% CI 1.09-1.37; $P<0.001)$ and the NPI carer distress rating
$(\mathrm{HR}=1.14,95 \mathrm{CI} 1.02-1.28 ; P=0.03)$ were both associated with an increased risk of admission to hospital. People with more advanced dementia based on CDR score were not more likely to be admitted to hospital than people with milder dementia $\left(P_{\text {trend }}=0.45\right)$. The CDR sum of boxes was similarly not statistically significantly associated with hospital admission. ${ }^{20}$

\section{Multivariable model}

Constructing a multivariable model using forward conditional stepwise entry highlighted NPI score as the only statistically significant independent predictor of admission to hospital (per s.d. disadvantage $\mathrm{HR}=1.21,95 \%$ CI 1.08-1.36; $P=0.001$; based on 251 admissions out of 681 individuals).

\section{Further analyses}

To examine the association between NPI score and hospital admission in more detail, we divided NPI scores into three groups based on recommended thresholds: those scoring 0 (the referent), 1 to 14 and greater than or equal to $15 .^{27}$ There was a doseresponse association between increasing NPI score and hospital admission ( $v$. NPI score of 0: NPI score $1-14 \mathrm{HR}=1.29,95 \%$ CI $0.95-1.76$; NPI score $\geqslant 15 \mathrm{HR}=1.82,95 \%$ CI $1.30-2.56$; $P_{\text {trend }}=0.002$ ), as shown in Fig. 2. Examining individual neuropsychiatric symptoms (NPI items) in a post hoc multivariable model using forward stepwise conditional entry highlighted agitation (per s.d. increase $\mathrm{HR}=1.28,95 \%$ CI 1.14-1.43; 
Table 2 Age- and gender-adjusted hazard ratios ( $95 \%$ confidence intervals) for the association between baseline characteristics and subsequent admission to hospital for any reason: Iongitudinal analysis of 730 men and women from the Scottish Dementia Research Interest Register

\begin{tabular}{|c|c|c|c|c|}
\hline & $N$ hospitalised & $N$ total & $\mathrm{HR}(95 \% \mathrm{Cl})$ & $P$ \\
\hline \multicolumn{5}{|l|}{ Diagnostic subtype } \\
\hline AD $v$. all others & 274 & 730 & $0.86(0.67-1.11)$ & 0.24 \\
\hline Mixed dementia $v$. all others & 274 & 730 & $0.86(0.61-1.22)$ & 0.41 \\
\hline Vascular dementia $v$. all others & 274 & 730 & $1.21(0.84-1.75)$ & 0.31 \\
\hline 'Other dementia'a $v$. all others & 274 & 730 & $1.67(1.09-2.56)$ & 0.02 \\
\hline Vascular risk factors ${ }^{b}$ & 274 & 730 & $1.03(0.81-1.30)$ & 0.82 \\
\hline Any comorbidity ${ }^{c}$ & 274 & 730 & $1.28(1.00-1.65)$ & 0.05 \\
\hline Having any carers $v$. none & 274 & 730 & $1.03(0.64-1.64)$ & 0.91 \\
\hline Prescribed cholinesterase inhibitor & 274 & 730 & $0.87(0.67-1.14)$ & 0.32 \\
\hline Prescribed antipsychotic & 274 & 730 & $1.32(0.77-2.27)$ & 0.31 \\
\hline Prescribed potentially inappropriate medication ${ }^{d}$ & 274 & 730 & $1.24(0.82-1.88)$ & 0.30 \\
\hline Mini-mental state examination ${ }^{\mathrm{e}}$ & 263 & 686 & $1.00(0.88-1.14)$ & 0.97 \\
\hline Instrumental Activities of Daily Living Scale $\mathrm{e}^{\mathrm{e}}$ & 235 & 663 & $1.08(0.95-1.22)$ & 0.26 \\
\hline Physical Self-Maintenance Scale $e^{e}$ & 259 & 701 & $1.18(1.04-1.33)$ & 0.01 \\
\hline Neuropsychiatric Inventory ${ }^{\mathrm{e}}$ (total) & 257 & 696 & $1.22(1.09-1.37)$ & $<0.001$ \\
\hline Neuropsychiatric Inventorye (carer distress) & 257 & 696 & $1.14(1.02-1.28)$ & 0.03 \\
\hline Clinical Dementia Rating Scale, overall rating & 259 & 699 & & 0.45 (for trend) \\
\hline 1 v. $0 / 0.5$ & & & $1.06(0.81-1.40)$ & \\
\hline $2 / 3$ v. $0 / 0.5$ & & & $1.24(0.89-1.72)$ & \\
\hline Clinical Dementia Rating Scale, sum of values ${ }^{e}$ & 251 & 677 & $1.04(0.92-1.17)$ & 0.57 \\
\hline \multicolumn{5}{|c|}{$\begin{array}{l}\text { a. 'Other dementia' comprises dementia with Lewy bodies, Parkinson's disease dementia, frontotemporal dementia, and other (not specified). } \\
\text { b. Vascular risk factors include hypertension, hypercholesterolaemia, diabetes and smoking. } \\
\text { c. Comorbidity includes cancer, respiratory disease, and diabetes. } \\
\text { d. Potentially inappropriate medication according to updated Beers criteria. }{ }^{22} \\
\text { e. Standardised so that hazard ratio is per standard deviation disadvantage. }\end{array}$} \\
\hline
\end{tabular}

$P<0.001)$ as the most important aspect of the NPI in terms of predicting admission to hospital.

Data were missing for one or more variables in $21.1 \%$ of the sample $(n=154)$. People with missing data were slightly younger, more likely to be female, less likely to have vascular risk factors but more likely to have a comorbidity of any sort, were more likely to have no carer and to have had more severe dementia, according to CDR and MMSE, but scored slightly better on IADL, PSMS and both scales of the NPI (online Table DS2). Thus, individuals with

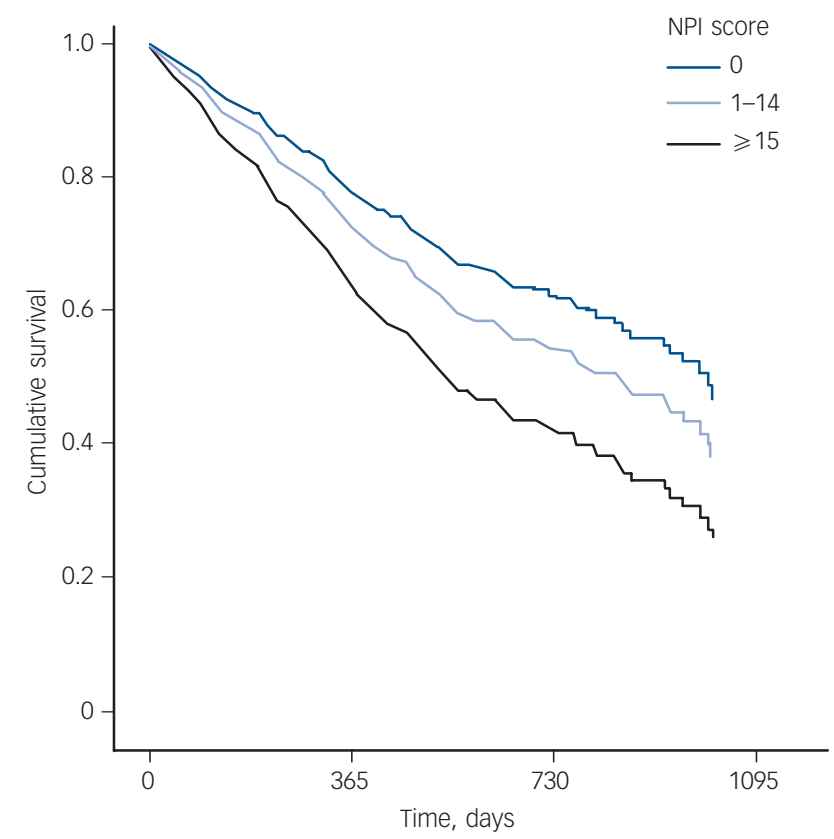

Fig. 2 Kaplan-Meier curves for time to general hospital admission in people with dementia stratified by Neuropsychiatric Inventory (NPI) score. missing data did not uniformly have less favourable levels of risk factors. Using multiple imputation to account for missing data in the multivariable models did not alter our conclusions (per s.d. disadvantage in NPI score $\mathrm{HR}=1.20,95 \%$ CI 1.07-1.35; $P=0.003$; 'other dementia' $\mathrm{HR}=1.56, \quad 95 \%$ CI $1.02-2.40$; $P=0.043)$.

\section{Discussion}

Our main finding was that a person with dementia being admitted to hospital over a mean of 1.2 years was predicted by their total NPI score, with risk increasing as category of severity rises. Further analyses suggested that agitation was the most important predictor within the NPI scale. Among the modifiable factors associated with dementia, bivariate analyses identified a relationship between risk of admission and comorbidity, carer distress and self-care, but these relationships did not persist in multivariable analyses. The risk of admission was significantly lower in the low and middle categories of NPI score than the highest category.

Observing this association gives us little information about the possible mechanism. One could speculate that more neuropsychiatric symptoms at baseline could reflect a higher burden of physical illness. Alternatively, the exacerbation of these symptoms, when already present, might be a common trigger for a general practitioner to seek admission. Whatever the mechanism, these symptoms are potentially modifiable and the important question is raised whether modifying them reduces unscheduled hospital admissions.

Neuropsychiatric symptoms are often important predictors of nursing home admission, ${ }^{29}$ and some consideration of these findings may be helpful. Persistent agitation or aggression early in dementia diagnosis may be associated with subsequent depressive symptoms in caregivers. ${ }^{30}$ Furthermore, time-varying measures of caregiver burden fully mediated the relationship between four behavioural disturbances (episodes of combativeness, 
property destruction, repetitive questions and reliving the past) and nursing home admission. ${ }^{31}$ Caregivers who did not indicate a care recipient's dangerous behaviour initially but did so subsequently (an 'incident' behaviour problem) were more likely to experience increases in burden $(P<0.0026) .{ }^{32}$ Caregivers who indicated greater emotional stress, a desire for the care recipient to enter institutional care and feelings of being 'trapped' in care responsibilities were more likely to admit people with dementia to nursing homes. However, demographic variables, incontinence and service use did not consistently predict nursing home admission. ${ }^{33}$

Thus, there are important parallels with factors that influence admission to care homes, particularly as behavioural changes in people with dementia are common and persistent. ${ }^{34}$ Our data are in keeping with the hypothesis that persistence of behaviour problems increases the risk of home placement breaking down and point to focusing on interventions reducing neuropsychiatric symptoms, especially agitation, which is among the most persistent of these problems, as those are potentially likely to decrease the risk of a person with dementia being admitted to a general hospital.

People with dementia are frequently admitted to hospital, ${ }^{2}$ and the issue of people with dementia in the general hospital is an important one; ${ }^{6}$ where possible, unscheduled admissions should be avoided in this patient group. ${ }^{7,8}$ However, it is surprising that, despite growing recognition of the public health importance of dementia, particularly in the setting of the general hospital, just over half of these people with confirmed dementia had their diagnosis correctly recorded on discharge from hospital. Recognising that a patient newly admitted to the general hospital has dementia is vital, perhaps most importantly as these people are at high risk of developing delirium which may be partially preventable, but also because the process of discharge planning should be informed by their diagnosis. ${ }^{2}$

Rarer forms of dementia, including frontotemporal lobar degeneration, are often associated with more prominent neuropsychiatric symptoms. This group was more likely to be admitted to hospital during follow-up in the bivariable models, but it seems that it might be the NPI score itself rather than diagnostic group per se which is driving this association. Although the mean NPI score was higher in the 'other dementia' category (17.9, s.d. $=17.8, v .10 .9$, s.d. $=15.1 ; P=0.012)$ the maximum scores were much higher in the Alzheimer's disease, mixed and vascular dementia group (105 points $v .61$ points). This is confirmed by the result of the Cox regression model including both the 'other' diagnostic category and total NPI score - only total NPI score remained statistically significant at conventional levels.

\section{Comparison with other studies}

Dementia is associated with an increased risk of hospital admission but few studies have investigated the impact of individual characteristics of people with dementia on their risk of admission. ${ }^{8}$ A recent systematic review of risk factors for people with dementia being admitted to hospital, comprising ten studies, reported that behavioural disturbance was associated with an increased risk of admission. ${ }^{9}$ The results of our study echo this and our finding that agitation may be the most important symptom is consistent with the findings reported in this systematic review.

There is little published research prospectively studying cohorts of people with dementia to identify baseline risk factors for admission to hospital. An American teaching hospital cohort study of 827 men and women with Alzheimer's disease found that $66 \%$ were admitted to hospital at least once over a median follow-up period of 3 years, compared with $41 \%$ over a mean
1.2 years in our study. ${ }^{10}$ They identified five independent risk factors: higher comorbidity, previous hospital admission, older age, male gender and shorter duration of dementia. These are similar to our findings apart from the effect of age (details of illness duration and previous admission to hospital were not available on the SDRIR), but they did not include measures of neuropsychiatric symptoms or function. The Réseau sur la Maladie d'Alzheimer Français (REAL.FR) study followed 686 patients with Alzheimer's disease from all over France for 2 years and found that $29 \%$ were admitted to hospital at least once in that period. ${ }^{11,13,14}$ They identified three independent risk factors for hospital admission: functional impairment, polypharmacy and greater NPI score. ${ }^{11}$ However, they did not present data on antipsychotic or inappropriate medication use within the polypharmacy risk they identified. Another French study following 134 patients with Alzheimer's disease from a memory clinic found that $23 \%$ were admitted to hospital in the following year. ${ }^{12}$ They identified that an inability to bathe independently and lower educational attainment were independent risk factors for admission to hospital, but had limited data on comorbidities and medication, and a small, unrepresentative sample.

\section{Strengths and limitations}

This is the largest general population prospective study of risk factors for hospital admission in dementia resulting in adequate power to detect fairly small effect sizes, including identifying a dose-response association between NPI score and hospital admission. A post hoc power calculation suggested that our sample size was powered to detect hazard ratios of approximately 1.2 with $80 \%$ power and a significance level of 0.05 . It was possible to follow these individuals up for up to 3.3 years during which period a large number were admitted to acute hospitals. The diagnostic categories were based on clinical diagnoses and were independently verified by two clinicians and so are likely to be robust. These people were at a relatively early stage of their illness at baseline, as demonstrated by the MMSE and CDR scores, and all were then community residents, so it may not be possible to extrapolate these results to all people diagnosed with dementia. However, the fact that this sample consisted mainly of people with mild to moderate dementia does not make the results any less important. The supplementary analysis examining individual NPI items was not specified a priori, but the fact that it supports findings from a previous systematic review gives us some confidence in this result. ${ }^{9}$

Since the hospital admissions were identified from national surveillance data-sets, it is likely that all hospital admissions were identified, that these data are robust, and thus the times to admission or censoring from entry to the register are accurate. The mean time to admission was 0.8 years and so the symptoms might not have still been present at the time of admission. However, neuropsychiatric symptoms have been shown to be relatively persistent, for example up to $67 \%$ for agitation over a period of 2 years. ${ }^{35}$ Comprehensive baseline data were available for participants, collected in a standardised manner by trained, skilled clinical studies officers from the Scottish Dementia Clinical Research Network. Furthermore, item-level data were available, allowing us to examine, for example, individual NPI items. The findings from the models using multiple imputation were similar to the main results, suggesting that missing data did not have a substantial effect on our conclusions.

\section{Implications and further research}

This study investigated a common and important condition and an important setting - the general hospital. Indeed, people with 
dementia in general hospitals were one of the main foci of the first Scottish Dementia Strategy. ${ }^{36}$ Our data were drawn from across Scotland, which avoids any problems with factors affecting hospital admission at a regional level such as local policies. Although the sample covers much of the country, it is not representative of the general population of people with dementia. Since these individuals are selected by expressing an interest in participating in dementia research, it is likely that they are healthier than the general population of people with dementia. This will affect the generalisability of our findings, but it is likely that conclusions drawn from studying this community-based sample can be broadly applied to the wider population of people with dementia.

If we accept our finding that total NPI score predicts acute general hospital admission in people with dementia, careful consideration is needed regarding the implications of this. Further research is needed to identify whether these admissions could be avoidable through interventions to treat neuropsychiatric symptoms. If it is the case that interventions to reduce neuropsychiatric symptoms and thus NPI score also reduce admission rates to hospital in this vulnerable group, many of the adverse consequences for people with dementia of being in hospital might be avoided. Our results suggest that such interventions should be targeted at individuals with higher levels of neuropsychiatric symptoms (NPI score $\geqslant 15$ ). Moreover, because neuropsychiatric symptoms correlate positively with carer strain, ${ }^{37}$ interventions that reduce neuropsychiatric symptoms might shorten admissions since carers under less strain might be more willing to accept discharge back home. Furthermore, risk stratification tools to predict unplanned hospital admissions, ${ }^{38}$ for example those used in the National Health Service in England \& Wales, do not currently incorporate mental health variables. Our results suggest that, at least in people with dementia, these variables may be among the most important in predicting unscheduled hospital admissions.

Clinical trials to examine whether a reduction in neuropsychiatric symptoms in people with dementia reduces unscheduled hospital admissions are now warranted. If this proved to be a modifiable risk factor for such admissions, it could have substantial clinical and public health impact.

Tom C. Russ, PhD, MRCPsych, Alzheimer Scotland Dementia Research Centre, University of Edinburgh, and Scottish Dementia Clinical Research Network, National Health Service (NHS) Scotland, Murray Royal Hospital, Perth, and Centre for Cognitive Ageing \& Cognitive Epidemiology, University of Edinburgh, and Division of Psychiatry, University of Edinburgh; Mario A. Parra, PhD, Alzheimer Scotland Dementia Research Centre, University of Edinburgh, and Scottish Dementia Clinical Research Network, NHS Scotland, Murray Royal Hospital, Perth, and Centre for Cognitive Ageing \& Cognitive Epidemiology, University of Edinburgh, UK, and UDP-INECO Ageing \& Cognitive Epidemiology, University of Edinburgh, UK, and UDP-INECO
Foundation Core on Neuroscience (UIFCON), Diego Portales University, Santiago, Chile; Alison E. Lim, Alzheimer Scotland Dementia Research Centre, University of Edinburgh; Emma Law, RMN, MPH, Peter J. Connelly, MD, FRCPsych, Scottish Dementia Clinical Research Network, NHS Scotland, Murray Royal Hospital, Perth; John M. Starr, PhD, FRCPEd, Alzheimer Scotland Dementia Research Centre, University of Edinburgh, and Scottish Dementia Clinical Research Network, NHS Scotland, Murray Royal Hospital, Perth, and Centre for Cognitive Ageing \& Cognitive Epidemiology, University of Edinburgh, UK

Correspondence: Dr Tom C. Russ, Division of Psychiatry, Kennedy Tower, Royal Edinburgh Hospital, Morningside Terrace, Edinburgh EH10 5HF, UK. Email: tc.russ@ed.ac.uk

First received 28 Feb 2014, final revision 1 Jul 2014, accepted 8 Jul 2014

\section{Funding}

Currently employed by the University of Edinburgh and NHS Lothian, from 2009 to 2013 , T.C.R. was supported by Alzheimer Scotland. T.C.R., M.A.P., A.E.L. and J.M.S. are members of the Alzheimer Scotland Dementia Research Centre at the University of Edinburgh. T.C.R M.A.P. and J.M.S. are members of the University of Edinburgh Centre for Cognitive Ageing \&.A.P. and J.M.S. are members of the University of Edinburgh Centre for Cognitive Ageing (G0700704/84698); funding from the Biotechnology and Biological Sciences Research
Council, the Engineering and Physical Sciences Research Council, the Economic and Social Research Council and the Medical Research Council is gratefully acknowledged for the latter. M.A.P.'s work is supported by Alzheimer's Society grant AS-R42303. The Scottish Dementia Clinical Research Network is funded by the Chief Scientist Office of the Scottish Government.

\section{References}

1 Luengo-Fernandez R, Leal J, Gray A. Dementia 2010: The Economic Burden of Dementia and Associated Research Funding in the United Kingdom. A Report Produced by the Health Economics Research Centre, University of Oxford for the Alzheimer's Research Trust. Alzheimer's Research Trust, 2010.

2 Russ TC, Shenkin SD, Reynish E, Ryan T, Anderson D, MacLullich AM. Dementia in acute hospital inpatients: the role of the geriatrician. Age Ageing 2012; 41: 282-4.

3 Andersen $\mathrm{K}$, Lolk A, Martinussen T, Kragh-Sorensen P. Very mild to severe dementia and mortality: a 14-year follow-up - the Odense study. Dement Geriatr Cogn Disord 2010; 29: 61-7.

4 Larson EB, Shadlen MF, Wang L, McCormick WC, Bowen JD, Teri L, et al. Survival after initial diagnosis of Alzheimer disease. Ann Intern Med 2004; 140: 501-9.

5 Rait G, Walters K, Bottomley C, Petersen I, Iliffe S, Nazareth I. Survival of people with clinical diagnosis of dementia in primary care: cohort study. BMJ 2010; 341: c3584.

6 Working Group for Liaison Mental Health Services for Older People, Faculty of Old Age Psychiatry, Royal College of Psychiatrists. Who Cares Wins. Improving the Outcome for Older People Admitted to the General Hospital: Guidelines for the Development of Liaison Mental Health Services for Older People. Royal College of Psychiatrists, 2005.

7 Lyketsos CG. Prevention of unnecessary hospitalization for patients with dementia: the role of ambulatory care. JAMA 2012; 307: 197-8.

8 Phelan EA, Borson S, Grothaus L, Balch S, Larson EB. Association of incident dementia with hospitalizations. JAMA 2012; 307: 165-72.

9 Toot S, Devine M, Akporobaro A, Orrell M. Causes of hospital admission for people with dementia: a systematic review and meta-analysis. J Am Med Dir Assoc 2013; 14: 463-70.

10 Rudolph JL, Zanin NM, Jones RN, Marcantonio ER, Fong TG, Yang FM, et al. Hospitalization in community-dwelling persons with Alzheimer's disease: frequency and causes. J Am Geriatr Soc 2010; 58: 1542-8.

11 Voisin $\mathrm{T}$, Andrieu $\mathrm{S}$, Cantet $\mathrm{C}$, Vellas $\mathrm{B}$. Predictive factors of hospitalizations in Alzheimer's disease: a two-year prospective study in 686 patients of the REAL.FR study. J Nutr Health Aging 2010; 14: 288-91.

12 Andrieu S, Reynish E, Nourhashemi F, Shakespeare A, Moulias S, Ousset PJ, et al. Predictive factors of acute hospitalization in 134 patients with Alzheimer's disease: a one year prospective study. Int J Geriatr Psychiatry 2002; 17: 422-6.

13 Voisin T, Sourdet S, Cantet C, Andrieu S, Vellas B. Descriptive analysis of hospitalizations of patients with Alzheimer's disease: a two-year prospective study of 686 patients from the REAL.FR study. J Nutr Health Aging 2009; 13: $890-2$.

14 Gillette-Guyonnet S, Nourhashemi F, Andrieu S, Cantet C, Micas M, Ousset P, et al. The REAL.FR research program on Alzheimer's disease and its management: methods and preliminary results. J Nutr Health Aging 2003; 7: 91-6.

15 Law E, Connelly PJ, Randall E, MCNeill C, Fox HC, Parra MA, et al. Does the Addenbrooke's Cognitive Examination - Revised add to the Mini-Mental State Examination in established Alzheimer disease? Results from a national dementia research register. Int J Geriatr Psychiatry 2013; 28: 351-5.

16 Mioshi E, Dawson K, Mitchell J, Arnold R, Hodges JR. The Addenbrooke's Cognitive Examination Revised (ACE-R): a brief cognitive test battery for dementia screening. Int J Geriatr Psychiatry 2006; 21: 1078-85.

17 Lawton M, Brody E. Assessment of older people: self-maintaining and instrumental activities of daily living. Gerontologist 1969; 9: 179-86.

18 Cummings JL, Mega M, Gray K, Rosenberg-Thompson S, Carusi DA, Gornbein J. The Neuropsychiatric Inventory comprehensive assessment of psychopathology in dementia. Neurology 1994; 44: 2308-14.

19 Hughes CP, Berg L, Danziger WL, Coben LA, Martin RL. A new clinical scale for the staging of dementia. Br J Psychiatry 1982; 140: 566-72.

20 Morris JC. The Clinical Dementia Rating (CDR): current version and scoring rules. Neurology 1993; 43: 2412-4.

21 Folstein MF, Folstein SE, McHugh PR. 'Mini-Mental State': a practical method for grading the cognitive state of patients for the clinician. J Psychiatr Res 1975; 12: 189-98. 
22 Connor DJ, Sabbagh MN, Cummings JL. Comment on administration and scoring of the Neuropsychiatric Inventory in clinical trials. Alzheimers Dement 2008; 4: 390-4.

23 Campanelli CM. American Geriatrics Society updated Beers Criteria for potentially inappropriate medication use in older adults. J Am Geriatr SoC 2012; 60: 616-31.

24 Cox DR. Regression models and life-tables. J R Stat Soc Ser B Stat Methodol 1972; 34: 187-220.

25 Lopez OL, Becker J, Wahed A, Saxton J, Sweet R, Wolk D, et al. Long-term effects of the concomitant use of memantine with cholinesterase inhibition in Alzheimer disease. J Neurol Neurosurg Psychiatry 2009; 80: 600-7.

26 Banerjee S. The Use of Antipsychotic Medication for People With Dementia: Time for Action. A Report for the Minister of State for Care Services. Department of Health, 2009.

27 Ballard CG, Thomas A, Fossey J, Lee L, Jacoby R, Lana MM, et al. A 3-month randomized, placebo-controlled, neuroleptic discontinuation study in 100 people with dementia: the neuropsychiatric inventory median cutoff is a predictor of clinical outcome. J Clin Psychiatry 2004; 65: 114-9.

28 World Health Organization. International Statistical Classification of Diseases and Related Health Problems, 10th Revision (ICD-10). WHO, 2010.

29 Zuidema SU, Derksen E, Verhey FRJ, Koopmans RTCM. Prevalence of neuropsychiatric symptoms in a large sample of Dutch nursing home patients with dementia. Int J Geriatr Psychiatry 2007; 22: 632-8.

30 Ornstein KA, Gaugler JE, Devanand DP, Scarmeas N, Zhu CW, Stern Y. Are there sensitive time periods for dementia caregivers? The occurrence of behavioral and psychological symptoms in the early stages of dementia Int Psychogeriatr 2013; 25: 1453-62.

31 Gaugler JE, Wall MM, Kane RL, Menk JS, Sarsour K, Johnston JA, et al. Does caregiver burden mediate the effects of behavioral disturbances on nursing home admission? Am J Geriatr Psychiatry 2011; 19: 497-506.

32 Gaugler JE, Wall MM, Kane RL, Menk JS, Sarsour K, Johnston JA, et al. The effects of incident and persistent behavioral problems on change in caregiver burden and nursing home admission of persons with dementia. Med Care 2010; 48: 875-83.

33 Gaugler JE, Yu F, Krichbaum K, Wyman JF. Predictors of nursing home admission for persons with dementia. Med Care 2009; 47: 191-8.

34 Aalten $\mathrm{P}$, de Vugt ME, Jaspers N, Jolles J, Verhey FR. The course of neuropsychiatric symptoms in dementia. Part I: findings from the two-year Iongitudinal Maasbed study. Int J Geriatr Psychiatry 2005; 20: 523-30.

35 Haupt M, Kurz A, Janner M. A 2-year follow-up of behavioural and psychological symptoms in Alzheimer's disease. Dement Geriatr Cogn Disord 2000; 11: 147-52.

36 Scottish Government. Scotland's National Dementia Strategy. Scottish Government, 2010.

37 Bradshaw LE, Goldberg SE, Schneider JM, Harwood RH. Carers for older people with co-morbid cognitive impairment in general hospital: characteristics and psychological well-being. Int J Geriatr Psychiatry 2013; 28: 681-90.

38 Academy Health. 2008 HSR Impact Awardee: Improving the Financing and Delivery of Health Care with Risk-Based Predictive Modeling. Academy Health, 2008.

\section{psychiatry in the movies}

\section{Wes Anderson's The Grand Budapest Hotel}

\section{Roxanne Keynejad}

I never lost my childhood awe of the author's creative power to craft characters from pure imagination. Perhaps this is why | chose psychiatry: the personalities and experiences of each patient remain far more vivid than anything I could think up. Wes Anderson, often lauded for his novel approach to film-making, challenges the perceived originality of creative genius in his latest film, The Grand Budapest Hotel. Speaking of his success as the fictional Republic of Zubrowka's national hero, Tom Wilkinson's 'The Author' confesses at the outset that his much-loved classic is pinched from mundane reality.

So begins an exhilarating cinematic adventure, told through chapters of The Author's famed novel, inspired by the tale related to his younger self (Jude Law), of immigrant lobby boy Zero Moustafa (the twinklingly melancholy F. Murray Abraham, eternally the Salieri of 1984's Amadeus for most of us).

Wes Anderson is not for everyone. His ensemble cast set pieces, from The Royal Tenenbaums to The Life Aquatic with Steve Zissou, can seem self-satisfied. Cramming in so many famous faces can detract from the interest invested in each character, decorated with superficial quirks, rather than developing their stories in depth. With the right screenplay, however, this approach can be his films' greatest success. Never was this truer than in The Grand Budapest Hotel, whose doll's house set design and chaptered plot lend themselves to relating brief, transient episodes in multiple characters' intertwining lives.

Despite the brevity of each star actor's turn, Anderson succeeds in challenging many cinematic clichés. He transforms one of our most reserved thespians, Ralph Fiennes, into campily comic concierge, M. Gustave. Filming on a tight schedule, with the entire cast staying together, coupled with his characteristic cinematography and set design, achieves the sense of attending a theatre company production. By casting well-known actors against type, from Willem Defoe to Tilda Swinton, Anderson seems to suggest that we may all be playing temporary roles and might just as easily wake up one morning being someone else entirely.

The 80-year-old aristocrat Madame Céline Villeneuve Desgoffe und Taxis' romance, murder and contested legacy, a stolen painting, prison break and high-speed ski chase are set against the backdrop of impending war, threatening the tranquil alpine state's future. The once-grand hotel's sad decline into matt browns and oranges against which solitary guests dine, forms an allegory for the lost magic of post-war Europe.

The Author credits his fortune to a tale told by a lonely hotelier, alluding to the inspiration of Anderson's own screenplay by the writings of Austrian playwright and novelist Stefan Zweig (1881-1942). This charmingly escapist film offers a message that for all The Grand Budapest Hotel's apparent creativity, it too is pinched from reality - like, perhaps, all other works of fiction. So as we leave the cinema, returning to more mundane surroundings, we may take comfort from Anderson's proposition: that the real magic is where we are, right now. 